\title{
Creating Effectiveness Principles for Principles- Focused Developmental Evaluations in Health-Care Initiatives: Lessons Learned from Three Cases in British Columbia
}

\author{
Ihoghosa Iyamu, Mai Berger, Erika Ono, and Amy Salmon \\ Centre for Health Evaluation and Outcome Sciences (CHEOS) / \\ University of British Columbia, Vancouver, British Columbia, Canada
}

\begin{abstract}
Principles-focused developmental evaluation is an emergent method of evaluation that is increasingly achieving relevance in initiatives seeking to transform health-care delivery within complex-adaptive systems. Creating meaningful effectiveness principles is considered a crucial first step in setting up such evaluations. In this article, we describe four practical steps that we applied in defining effectiveness principles to align with Patton's GUIDE criteria. To illustrate our approach, this article features three principles-focused developmental evaluations implemented in British Columbia, highlighting lessons learned through the process of creating effectiveness principles.
\end{abstract}

Keywords: developmental evaluation, effectiveness principles, principles-focused evaluation, utilization-focused evaluation

Résumé : Lévaluation évolutive axée sur les principes est une nouvelle méthode d'évaluation qui se montre de plus en plus pertinente pour des projets visant à transformer la prestation de soins de santé au sein des systèmes adaptatifs complexes. La création de principes d'efficacité significatifs est considérée comme une première étape cruciale de la mise en place d'une telle évaluation. Dans le présent article, nous décrivons les cadres et pratiques que nous avons appliqués pour définir les principes d'efficacité en vue de nous conformer aux critères "GUIDE» de Patton. Pour illustrer notre approche, l'article présente trois évaluations évolutives axées sur les principes qui ont été effectuées en Colombie-Britannique. Il est question des leçons apprises dans le cadre du processus de création des principes d'efficacité.

Mots clés : évaluation évolutive, principes d'efficacité, évaluation axée sur les principes, évaluation axée sur l'utilisation

In this article, we reflect on our experiences of creating effectiveness principles in three principles-focused developmental evaluations that took place between

Corresponding author: Amy Salmon, Knowledge Translation Unit, Centre for Health Evaluation and Outcome Sciences (CHÉOS), 588-1081 Burrard Street, Vancouver, British Columbia V6Z 1Y6, Canada; asalmon@cheos.ubc.ca 
2018 and 2020 in British Columbia. First, we describe principles-focused developmental evaluations and the relevance of this approach in health-care contexts. Second, we present three cases, highlighting the features that made them suitable for principles-focused developmental evaluations, how effectiveness principles were created, and the challenges we encountered. Third, we describe four steps we each took in the process of applying Patton's (2018) GUIDE framework for creating effectiveness principles based on our experiences. Finally, we discuss collective lessons learned, focusing on how the creation of effectiveness principles can be useful for evaluators in health-care systems research. In so doing, we aim to contribute to emerging literature on principles-focused developmental evaluation in health-care settings by illustrating concrete and practical examples.

\section{BACKGROUND}

\section{Principles-focused evaluation and effectiveness principles}

Principles-focused evaluation (P-FE) is a novel evaluative approach developed by Michael Quinn Patton (2018). Originally applied in the context of developmental evaluation, P-FE has evolved into an evaluation approach unto itself with a specific and unique focus. While more traditional approaches consider the program or initiative itself (or its components) as the evaluand, a P-FE evaluates the meaningfulness of principles guiding the program, the process of implementing principles, the outcomes associated with the principles, and the broader, longlasting impacts of the principles when applied (Patton, 2018).

In the context of a P-FE, principles become the hypotheses that are examined and tested. Principles are values-based statements that inform and guide decisions and actions in different contexts. Principles also determine what is to be expected based on the actions that are taken. They can be evaluated in terms of their design (are the principles clear, meaningful, and actionable?), the process (was the principle followed?), and the outcome (was the required outcome accomplished when the principle was followed?). However, to be effective in the program or initiative, and to be appropriately evaluated in this way, these principles must be framed as effectiveness principles (Patton, 2018; Turner \& Cromhout, 2020). Effectiveness principles must be distinguished from moral principles, which guide our understanding of what actions are right or wrong, and from natural principles, which account for how the world works (Patton, 2018). More detailed descriptions of the types of principles and a discussion of their various applications can be found in Patton (2018).

While all principles are evaluable, effectiveness principles are useful for clarifying which actions can lead to specific results in specific contexts by describing actions in clear and meaningful ways. Effectiveness principles provide guidance for action in the face of complexity and have to be "interpreted and adapted to context" (Patton, 2018, p. 211). Therefore, P-FE is most suited to principles-driven programs or initiatives that are based on a need for action that is both evidenceand values-based. Patton (2018) offers the GUIDE framework as a set of criteria for creating and clarifying meaningful effectiveness principles (Figure 1). 


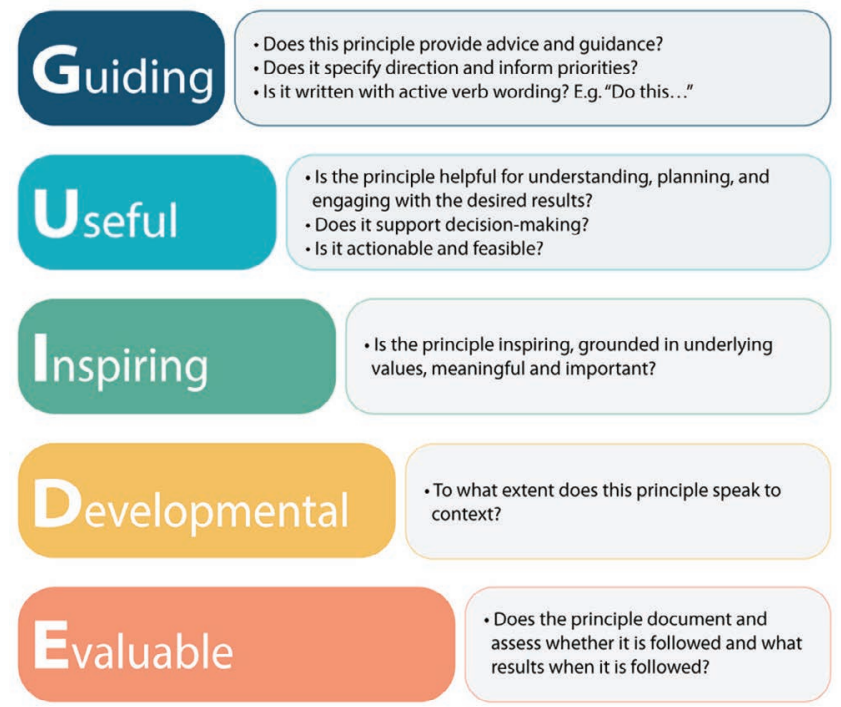

Figure 1. GUIDE acronym and mnemonic. GUIDE indicates that an effectiveness principle must (a) provide Guidance, (b) be Useful, (c) Inspire, (d) support ongoing Development, and (e) be Evaluable. Figure adapted from Patton (2018).

\section{Principles-focused developmental evaluation}

Principles-focused evaluations can simultaneously be developmental evaluations (DE) and a distinct evaluative approach called principles-focused developmental evaluation (P-FDE) (Patton, 2018). DE is intended to support evidence-informed decision making in the adaptive development of new models and approaches in the earliest stages of innovation, especially in contexts where outcomes are unpredictable or evidence and metrics regarding expected formative and summative outcomes is scarce (Dozois et al., 2010; Patton, 2018; Patton et al., 2015). Figure 2 describes the relationships between DE, P-FE, and P-FDE (adapted from Patton, 2018). All three of the evaluations presented in this paper were conducted as P-FDEs.

\section{Principles-focused developmental evaluations in health care}

P-FE and P-FDE approaches can be particularly useful in health care, especially for assessing programs or initiatives that are increasingly adopting principles promoting equity in service delivery and person-centredness to guide the process of health systems transformation (Miles \& Asbridge, 2013). This often requires health innovators to shift from "rules-based" approaches that have characterized health systems for decades to more principles-driven approaches. Such shifts allow health-care providers to have the flexibility to make choices about how to deliver services that prioritize the needs and rights of service users, while still maintaining equivalent levels of accountability (Turner \& Cromhout, 2020). Therefore, creating effectiveness principles to facilitate these P-FDEs can be a delicate but useful activity, as demonstrated in the cases presented. 


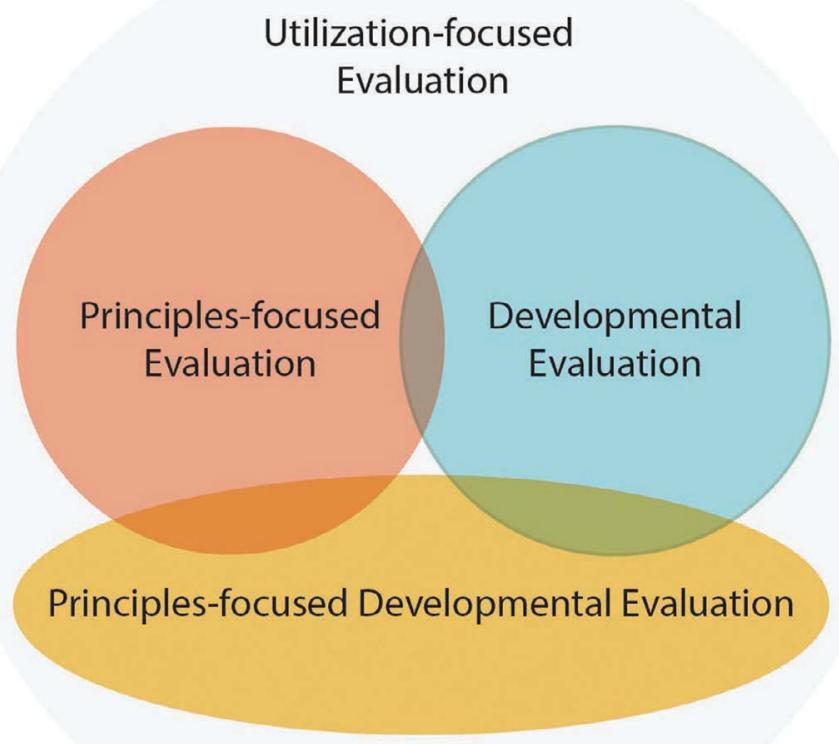

Figure 2. Relationships between utilization-focused evaluation, principles-focused evaluation and developmental evaluation. Figure adapted from Patton (2018).

While the literature is clear about the features of principles-focused evaluations and effectiveness principles, there is a dearth of information on how to translate these concepts into practice. Specifically, there is a lack of practical examples relevant to P-FDEs applied in health-care settings as compared to the literature on P-FDEs conducted in the contexts of community-based initiatives. Since P-FDEs typically begin with the development of effectiveness principles, we thought we should start there. In this article we seek to add clarity and greater precision to the GUIDE framework by providing a condensed, four-step process of creating effectiveness principles, with concrete examples from our experience and learnings from three developmental evaluations in transformative health programs. These steps may be considered as a consolidation of Patton's 10-step checklist (Patton, 2018, pp. 348-350) and may be useful in framing the ongoing activities of the evaluation. The four steps we discuss are as follows: engagement with stakeholders; reviewing underlying theories, values, existing research, and available data; drafting candidate effectiveness principles; and reviewing and finalizing effectiveness principles using the GUIDE criteria. These steps have been derived from our collective critical reflection on experiences from the three cases. 


\section{THE THREE CASES}

Our discussion of the practical steps required to create effectiveness principles is situated in three P-FDEs of health-care initiatives that were carried out between 2018 and 2020. Although all three cases were unique, the initiatives shared similarities in their objective to transform health-care delivery within the complexadaptive systems in which they were implemented. Therefore, the evaluations had to attend to the complex environment of the initiatives and support their ongoing development. To conduct these P-FDEs, the evaluators embedded themselves within the program teams, participated in and observed implementation, and routinely shared emerging results to enable course corrections. Thus, ongoing learning was facilitated as implementers and evaluators gained clarity on the context, principles, and theories necessary to drive the initiatives. Note that while the initiatives were endeavouring to achieve transformation at the systems level, we situate the evaluations and analysis at the level of the intervention.

\section{Case 1: Inpatient psychiatry redevelopment (September 2018- March 2019)}

\section{Background}

The inpatient psychiatry redevelopment principles-focused developmental evaluation was conducted as part of the pilot project at the St. Paul's Hospital (SPH) redevelopment initiative, under Providence Health Care (PHC), which aims to relocate and build a new hospital in 2026. The redevelopment initiative was proposed to begin the operational planning phase with a pilot project focusing on the inpatient psychiatry units.

This pilot project was a collaborative undertaking by several PHC clinical support teams. The goal was to provide insights that would guide the inpatient psychiatry units in planning their future state at the new $\mathrm{SPH}$, as well as trialling a collaborative approach to health-care delivery planning that utilized process mapping, visioning exercises, patient engagement, data analytics, and principlesfocused developmental evaluation methods.

\section{Purpose}

The purpose of the inpatient psychiatry redevelopment P-FDE was to establish an inpatient psychiatry implementation plan describing the immediate, short-term, and long-term actions for the redevelopment initiative to achieve its future state. In addition, the redevelopment initiative aimed to create a standardized approach to engagement and evaluation for care delivery planning that could be used in other clinical programs across the hospital. A P-FDE approach was well suited to the values-driven and change-oriented nature of this initiative.

\section{Effectiveness principles development}

Identifying effectiveness principles involved several stages, including reviewing, validating, and updating their current state map; reviewing their model of care document; reviewing their standard key metrics (such as length of stay 
and alternative level of care); patient engagement; reviewing care processes and facilitating visioning exercises; and participating in and co-facilitating the SPH redevelopment initiative working group.

During these stages, data collection for establishing effective principles included, first, a textual analysis of the SPH redevelopment function plan, SPH redevelopment clinical specifications plan, and the inpatient psychiatry units' policies, procedures, and models of care. Second were reflection sessions during the SPH redevelopment initiative working group meetings. Third, over the span of four months, the evaluator co-facilitated seven focus group engagement sessions with nurses and allied health professions, a one-to-one interview with an inpatient psychiatry clinical nurse leader, and a one-to-one interview with the SPH patient and family engagement coordinator. Throughout this iterative process, candidate effectiveness principles were established during the first few focus groups with inpatient psychiatry service providers and were modified during the subsequent focus groups and interviews. By the end of the four months of data collection for creating the effectiveness principles, six effectiveness principles were finalized and agreed upon by staff, management, and the working group (Table 1).

\section{Case 2: Foundry's integrated step care model (February 2018- March 2020)}

\section{Background}

Foundry is a provincial initiative building a network of "one-stop shops" (called Foundry centres) for young people and their families to improve access to mental health and social services in communities across British Columbia. Currently,

Table 1. Inpatient psychiatry redevelopment effectiveness principles

Patient and staff safety: Make decisions about care that promote patient and staff safety.

Patient- and family-centered care: Match length of stay to patients' care needs; include families in patients' care and provide safe visiting space.

Trauma-informed: Recognize that many patients in psychiatry have experienced trauma-build responses, relationships, and services on that knowledge.

Recovery-oriented: Accept that recovery outcomes are personal and unique for each patient and go beyond an exclusive health focus to include an emphasis on social inclusion and quality of life. Provide care that supports a process of hope and healing.

Harm-reduction: Work with patients to reduce negative consequences associated with drug use. Respect patients' rights to define what their goals are, and do not assume that all patients aim for abstinence.

Integrated care, collaboration, and information sharing: Provide integrated responses through collaboration across PHC services and with partners in primary, tertiary, and community care. Provide assessments/consultations at the right time and place. 
there are 11 Foundry centres in operation across the province, and an additional eight are expected to open by the end of 2020. Each Foundry centre offers a core suite of services, including primary health-care services, mental health services, substance-use services, social services, and peer-navigation services, which are housed together. Foundry centres organize and monitor the delivery of services through clinical pathways for mood disorders, substance-use problems, and early/ first-episode psychosis that utilize a stepped care approach. At the time of this writing, a fourth pathway for disordered eating is also in development. Each pathway has four "steps," with each step representing a level of care that is based on the needs of the young people and their family or circle of care (defined as people whom young people identify as important in their treatment and care planning). Similar to many stepped-care models, the services are delivered in such a way that the most effective and least resource-intensive treatment is intended to be delivered first, and young people are "stepped up" along the clinical pathway to intensive / specialist services only as required. Due to the availability of diverse health services and stakeholders under one roof, services are offered along the clinical pathways in an integrative way and, therefore, comprise Canada's first integrated stepped care model (ISCM).

\section{Purpose}

Foundry Central Office commissioned our team to conduct an evaluation to assess and support the early implementation and adaptive development of Foundry's ISCM. Our approach for the evaluation of Foundry's ISCM was based on a previously completed DE of Foundry's proof-of-concept period (Salmon et al., 2018). During the proof-of-concept DE, we came to understand that Foundry is best understood as a complex adaptive system of care for young people and their families, where many of the conditions change substantially over time and are not entirely within the Foundry's locus of control (Salmon et al., 2018). This understanding was particularly relevant to the ISCM implementation, where, for example, the heterogeneity of the service-provision landscapes in each of the communities could result in differences in how each Foundry centre operationalized the pathways. Therefore, conventional evaluation frameworks guided by more traditional theories of change would not reflect each Foundry centre's adaptive development of the ISCM to their context and fail to capture useful information for the involved stakeholders and program planners.

Our P-FDE of Foundry's ISCM was intended to be responsive to the complex environment in which Foundry and Foundry centres operate, allowing for early results to be examined, and enable any needed course corrections before the ISCM was fully consolidated and brought to scale. Our aim with this evaluation was to identify and establish core effectiveness principles for the ISCM, to determine whether these effectiveness principles are being followed in the development and implementation of ISCM, to help stakeholders to navigate the uncertainties and emerging challenges they face during implementation, and to assess whether maintaining fidelity to these principles is leading to desired results. Overall, the 
analysis of the implementation and adaptive development of Foundry's ISCM was intended to provide much-needed evidence for how to implement up-stream prevention and recovery-oriented systems of care for young people and their families.

\section{Effectiveness principles development}

The first step in the ISCM evaluation was to develop and consolidate candidate effectiveness principles. This was accomplished in the following manner. First, a list of candidate effectiveness principles was drafted by the Principal Evaluator (Salmon), based on the GUIDE framework, a review of existing documentation produced by Foundry about the ISCM, and in consultation with Foundry's director of clinical operations (who created the ISCM) and Provincial Clinical Working Group (composed of representatives with clinical and operational decision-making authority in each community or region with a Foundry centre). Next, the candidate effectiveness principles were workshopped with members of the Foundry Central Office team, front-line mental health and substance-use clinicians and leadership at each of the three participating Foundry centres, and with Foundry's Provincial Youth Advisory Council. These workshop sessions occurred in meetings ranging from 1.5 to 2 hours in length and were held from December 2018 to February 2019. Like a world café, each workshop session built iteratively off the contributions of the group preceding it.

After the workshopping was complete, the revised candidate effectiveness principles were once again brought back to the Provincial Clinical Working Group for their discussion and an agreement to proceed with the effectiveness principles in their current form, such that the candidate effectiveness principles would form the hypotheses guiding the evaluation. Using the candidate effectiveness principles, we created an evaluation matrix, in consultation with Foundry Central Office, which matched specific indicators and/or assessment modes to each effectiveness principle, as well as those related to general dimensions of integration, acceptability, and operational effectiveness described by Bower and Gilbody (2005) as necessary for the evaluation of stepped-care models. These dimensions and indicators were then assessed qualitatively through semi-structured interviews and ethnographic observations, and quantitatively by specific items and measures embedded into surveys for clients and their families or caregivers and regularly administered by Foundry. These analyses were supplemented by analyses of select client-level clinical and administrative data.

\section{Case 3: Megamorphosis (June 2019 to June 2020)}

\section{Background}

Megamorphosis is an ongoing initiative implemented by Providence Health Care (PHC) since 2017 as part of its "Home for Us" strategy. The initiative aims to improve the quality of life for the diverse population of long-term care (LTC) residents by shifting the culture from an institutional model to a relationshipcentred and resident-directed social model. To date, Megamorphosis has been 
implemented in six neighbourhoods of four of PHC's LTC homes in metro Vancouver, with the aim of facilitating the required culture shift.

The innovative approaches integrated in the Megamorphosis initiative were designed with learnings from an insights-gathering process that leveraged humancentred design principles to engage residents, families, and staff in its LTC homes. This insights-gathering process, which was completed between September and November 2014, helped to crystallize perspectives and priorities of care as expressed by LTC residents, their families, and staff. It also provided insights into the specific challenges that staff, families, and residents experienced in routine care, which informed the initiative's strategies. These strategies focus on increasing the engagement of each resident at the LTC through various activities. Through the process of an initial literature review, insights gathering, consultations with stakeholders, and focused-team discussions, three guiding values (initially referred to as principles), which drive the initiative, were identified: emotional connections matter most; residents direct each moment; and home is not a place, it is a feeling.

With a focus on experiential learning, the initiative has been implemented in a series of iterations at each LTC home. The iterations consist of three clearly outlined periods: Prework-six to eight weeks of preparatory activities such as stakeholder engagement, relationship-centred care training or experiential sessions and community gatherings to prepare the site for transformation; Intensive phase - two weeks of rapid cycle testing of ideas during which residents, families, and staff co-create and test changes together; and Sustainment-two to four weeks of community engagement to continuously reinforce and improve changes tested in the intensive phase.

\section{Purpose}

In June 2019, our team was commissioned to carry out an evaluation to support the process of evidence synthesis in the ongoing initiative. The key evaluation needs were to understand if and how Megamorphosis has supported a culture shift at the LTC home, with the aim of using the emerging evidence to inform future iterations of the initiative. This evidence was crucial, as PHC LTC leaders were readying Megamorphosis for scale-up but as yet had no standardized processes for facilitating culture change within their health systems. Considering that the initiative was in its nascent phase and aimed to create social innovation in the complex and dynamic LTC system through well-outlined principles, it was apparent that Megamorphosis would be well suited to a P-FDE from the outset. Furthermore, its iterative design and need for adaptive learning were well aligned with the utilization-focused characteristic of this evaluation approach.

\section{Effectiveness principles development}

As indicated earlier, Megamorphosis was built around three guiding values. These values were deeply integrated with the implementation framework. However, our initial assessment suggested that these values (called principles at the time) did not meet the GUIDE criteria for effectiveness principles (Patton, 2018). 
As the first step, the initiative's documents were reviewed and key informant interviews were conducted with front line staff, residents, families, and leaders at the site of the next iteration. These interviews sought to identify the baseline culture of care and the existing values guiding care. In addition, through interviews and extensive ethnographic observations, further insight was gained into the operational context, existing challenges, group dynamics and underlying norms relevant to the intended culture of care.

Thereafter, the evaluation assessed the supporting evidence grounding similar models of culture shift in relation to the Megamorphosis model being evaluated. The process included a rapid review of the evidence, analysis of the baseline data using grounded theory, and mapping the existing evidence to various components of the initiative. This provided a benchmark for collectively assessing the adequacy of the three values as the basis for the initiative. Thereafter, candidate effectiveness principles were drafted drawing from the data obtained through the interviews, observations, and rapid reviews. The drafted principles were aligned with the "guiding values," which continued to be entrenched in the model's framework. The effectiveness principles were then collaboratively appraised with the initiative's implementing team and other key stakeholders using the GUIDE framework. This informed the reframing of the substantive candidate principles (Table 2) to meet the GUIDE criteria, while being appropriate and resonant with the beneficiaries and stakeholders.

\section{A FOUR-STEP PROCESS FOR CREATING EFFECTIVENESS PRINCIPLES}

This article presents the process of formulating effectiveness principles, which is crucial for setting up a P-FE or P-FDE. Through a process of team reflection on and learning about each succeeding evaluation, we were better able to articulate, clarify, and target our practice. This aligns with the Canadian Evaluation Society's (CES) core competency domain of reflective practice, which emphasizes continuous learning and professional growth. As such, situational examples and reflections may be presented with a predilection to the more recent cases.

Through our experiences, we have come to understand that the process is iterative and non-linear, requiring flexibility on the part of the evaluation team and stakeholders. We have identified four distinct steps that are important in the process of creating high-quality effectiveness principles: engagement with stakeholders; review of underlying theories, values, existing research, and available data; drafting candidate effectiveness principles; and stakeholder review and finalization of effectiveness principles using Patton's (2018) GUIDE criteria. These steps can be iterative and embrace the rigour required of any principles-focused and developmental evaluation. The order in which the steps occur may differ depending on the needs and processes of individual evaluations. However, we find that from an evaluative standpoint, these distinct steps are crucial in the process of clearly articulating high-quality effectiveness principles (Patton, 2018). 
Table 2. Megamorphosis effectiveness principles

\begin{tabular}{|c|c|}
\hline Guiding principles & Effectiveness principles \\
\hline $\begin{array}{l}\text { Home is not a place, } \\
\text { it is a feeling }\end{array}$ & $\begin{array}{l}\text { Create and maintain a home-like environment for those who } \\
\text { live, visit, and work at our LTC homes. }\end{array}$ \\
\hline \multirow[t]{2}{*}{$\begin{array}{l}\text { Residents direct } \\
\text { each moment }\end{array}$} & $\begin{array}{l}\text { Empower staff to holistically respond to the needs of } \\
\text { residents in each moment by organizing supporting } \\
\text { systems around the residents' needs. }\end{array}$ \\
\hline & Respect residents' wishes regarding the care that they receive. \\
\hline \multirow{2}{*}{$\begin{array}{l}\text { Emotional } \\
\text { connections } \\
\text { matter most }\end{array}$} & $\begin{array}{l}\text { Create and maintain emotional connections between those } \\
\text { who live, visit, and work at our LTC homes. }\end{array}$ \\
\hline & $\begin{array}{l}\text { Enhance the dignity of all who live, visit, and work at our LTC } \\
\text { homes. }\end{array}$ \\
\hline \multirow[t]{3}{*}{$\begin{array}{l}\text { Human-centred } \\
\text { design }\end{array}$} & $\begin{array}{l}\text { Cultivate and sustain processes for self and team-reflection } \\
\text { and continuous improvement among those who live, visit, } \\
\text { and work at each neighbourhood. }\end{array}$ \\
\hline & $\begin{array}{l}\text { Understand challenges through collaborative processes that } \\
\text { engage those who live, visit, and work at our LTC homes. }\end{array}$ \\
\hline & $\begin{array}{l}\text { Generate and test solutions through shared governance } \\
\text { by involving those who live, visit, and work at each } \\
\text { neighbourhood. }\end{array}$ \\
\hline \multirow[t]{2}{*}{$\begin{array}{l}\text { Relationship- } \\
\text { centred care }\end{array}$} & $\begin{array}{l}\text { Promote and sustain open dialogue and emotional } \\
\text { connections within the multidisciplinary team. }\end{array}$ \\
\hline & Know each other's stories to support care as a relationship. \\
\hline
\end{tabular}

We also realize that applying systems thinking in each of the steps as described can be a useful strategy (Patton, 2011). Health systems involve complex, interrelated, and interdependent processes that must be considered at each step of effectiveness principles development, as described below (Cole et al., 2014; Wolfe, 2020). A systems lens allows the evaluation and implementation team to identify the full range of barriers, principles, and actors that need to be considered for the goals of the initiatives to be achieved (Patton et al., 2015). In some cases, these factors may exist outside the initiatives' originally defined boundaries.

\section{Engagement with stakeholders}

Similar to most evaluative approaches, engaging with stakeholders is an important step in a P-FDE (Centers for Disease Control and Prevention, 2011), which can launch the creation of effectiveness principles. Through systematic engagements, evaluators can work with stakeholders, particularly front-line leaders and patient/ client advisors, to clarify the evaluation's distinctive approach and its focus on principles as an evaluand (Patton, 2018). This is crucial to setting up the collaborative processes that can enhance stakeholders' understanding and foster ownership of the evaluation (Cole et al., 2014; Kinarsky et al., 2019).

As Patton (2018) asserts, it is important to make the distinction between effectiveness principles and natural or moral principles. Effectiveness principles are 
often informed by natural and/or moral principles but should be framed in a way that meets the GUIDE criteria (Patton, 2018). Through stakeholder engagements, the natural principles or guiding values can be clarified and differentiated from the effectiveness principles. In some cases, these principles-driven programs or initiatives may have identified goals and strategies based on implicit principles, and in other cases, they may already have clearly outlined guiding values.

The latter case may present significant challenges to the evaluation team. For example, in the evaluation of Megamorphosis, the team had already gone through a systematic process to develop their guiding principles. These principles were certainly inspiring and had become integral to the initiative's identity but did not meet the GUIDE criteria. It was apparent that upending the body of work in articulating guiding principles would be contentious for the initiative's team. To navigate this situation, we introduced the concept of effectiveness principles as an opportunity to rearticulate the existing principles in a clear, actionable, adaptable and evaluable manner, rather than as a replacement for the existing principles that were then reframed as "guiding values" (see Table 2).

In contrast, the Inpatient Psychiatry Redevelopment P-FDE had the task of creating effectiveness principles without any pre-defined principles but with implied organizational goals. However, developing effectiveness principles in collaboration with diverse stakeholder groups like the Redevelopment Initiative Working Group and the inpatient psychiatry units can be time-consuming and complex. With a six-month window to complete the project, it was crucial to collaboratively prioritize activities that could be completed in time. Therefore, the majority of the time for the P-FDE focused on building trusting relationships with stakeholders while identifying, generating, developing, and articulating effectiveness principles that were meaningful to both the redevelopment leadership and to the clinical teams. This is important because P-FDEs require a foundation of trust, subjective judgement, and vulnerability (Patton, 2018).

Furthermore, regular stakeholder meetings, implemented in each of the three case evaluations, help to ensure that both the effectiveness principles and evaluation remain focused on their utility for the intended users (Patton, 2016). From the outset of each P-FDE, we set up working groups, which consisted of a group of stakeholders who, in conjunction with the evaluation team, were responsible for steering the evaluation and ensuring the use of the information generated from the evaluation. Over time, these working groups collaboratively worked to arrive at the final effectiveness principles. This process aligns well with the principle of co-creation that facilitates the acceptability and use of the effectiveness principles (Patton, 2016, 2018; Patton et al., 2015).

\section{Review of underlying theories, values, existing research, and available data}

At this step, supporting evidence for the implicit or explicit values of the program or initiative can be appraised through literature review, document analysis, and other forms of textual analysis. Textual analysis can be particularly important for 
identifying underlying values and delineating the mechanisms through which they can affect the overall program or initiative and its intended results. Integrating primary-source data helps the evaluator test assumptions made by the team in the design of the program or initiative and can be helpful in identifying blind spots that may have been missed. This can inform further adaptation of the program or initiative. An extensive textual analysis process was conducted in the Inpatient Psychiatry Redevelopment P-FDE, due to the large volume of documents that were developed for the process of planning and building a new hospital campus. This P-FDE started data collection with both the working-group meetings and textual analysis to create the first set of candidate effectiveness principles, which were then adapted throughout the process of conducting focus groups and informant interviews.

Where appropriate, it may be beneficial to integrate ethnographic methods in the primary data-collection plan. Ethnographic approaches are an effective way to uncover the values that are tacitly expressed in the manner of interrelations of the social actors within their natural environment (Lambert et al., 1995). In each of the three cases, we conducted key informant interviews and focus-group discussions while drafting the candidate effectiveness principles. We found that this process helped to determine the priorities of the key stakeholders and ensured that the principles were reflective of the views of the key stakeholders from their initial formulation. Methods such as participant and non-participant observations can also provide opportunities to understand how candidate effectiveness principles are applied in practice. This is especially important for social change innovations in health care that are reliant on boundary partners to translate the effectiveness principles into actions that can result in the theorized outcomes. As previously stated, applying a systems lens to the initial review of theories, existing data, and description of themes can help the evaluator and the team to identify additional principles that facilitate the implementation of existing principles. Finally, collaboration with the stakeholders in this review can help answer remaining questions that designers of the program or initiative may have.

\section{Drafting candidate effectiveness principles}

Effectiveness principles should be drafted with key considerations from the GUIDE criteria (Patton, 2018). The criteria have been likened to the SMART criteria, which have been used to assess program or initiative goals and objectives (Turner \& Cromhout, 2020). The GUIDE criteria suggest that effectiveness principles must be

Guiding: to provide guidance, the principles should be worded with an imperative to action, especially in an expected direction. In this regard, they need to be prescriptive, clearly pointing the way to success, rather than just being statements of values. (Patton, 2018).

In Megamorphosis, the guiding principle - "Home is not a place, it is a feeling"succinctly captured the values of the initiative, yet it did not provide information 
or directions on how to achieve these values. By reframing the principle as "Create and maintain a home-like environment for those who live, visit, and work at our LTC homes," we were able to create a call to action.

The second part of the GUIDE acronym is

Useful: practical principles that can be applied in frequently encountered situations on the project can be considered useful. A useful principle is one that is clear, feasible, supportive in making sense of frequently encountered situations, and strikingly descriptive of how to be effective in these situations. To create useful principles, the evaluation team must rely deeply on the insight of the beneficiary communities and organizations that enact the principle.

For example, in the Inpatient Psychiatry Redevelopment P-FDE, all stakeholders in leadership and front-line roles agreed that "Patient and Staff Safety: Make decisions about care that promote patient and staff safety" was the most important and useful effectiveness principle. They all agreed that if any principles clashed during a specific encounter, decisions on how to act always came down to safety for patient and staff. They claimed that without this principle, the other principles could not be fully realized.

GUIDE continues:

Inspiring: As Patton (2018) described, principles need to draw on the deeper beliefs and values of the organization and the community to be effective. They must give meaning and be poignant in a way that evokes a sense of purpose amongst the social actors.

In our experience, baseline interviews provide rich descriptions of the deeply embedded organizational and community values. These descriptions are useful for creating candidate effectiveness principles with language that resonates with the organization and communities. For example, it was apparent from the baseline interviews on the Megamorphosis evaluation that respect for older adults was a culturally important value especially among the front-line staff. Therefore, reframing the value "Residents direct each moment" to include principles such as "Respect residents' wishes regarding the care that they receive" ensured that the principle tapped into the staff's closely held values. In the ISCM evaluation, we found that the use of participatory engagement processes with focus groups and workshops improved the "buy in" for the evaluation process among stakeholders, as these approaches ensured that the participants' own beliefs and values that motivate their work were reflected in the evaluands being crafted. These approaches are particularly relevant to implementation contexts with considerable heterogeneity (such as those we encountered with the Foundry ISCM and Megamorphosis evaluations), where stakeholders may have concerns about the uniqueness of their context being eclipsed in formative evaluations emphasizing fidelity to a system-wide model of approach.

The fourth component of GUIDE is

Developmental: In health-care settings, context is important and highly dynamic. In health-care contexts, leaders and innovators commonly need to be able to appraise 
situations and make implementation decisions that simultaneously activate the vision, mission, and values articulated at a system level while, at the same time, they respond to the dynamic and heterogenous contexts in which these implementations occur. Therefore, to be developmental, the effectiveness principles need to be written in a way that takes explicit account of the system complexities and can be adapted to each context and changing situations over long periods of time.

In all three case examples of evaluations discussed in this paper, we found that using participatory and inclusive approaches to crafting effectiveness principles reinforced the developmental aspects of the work being undertaken in two important ways. First, this approach to identifying and articulating effectiveness principles ensured that the experiential knowledge and perspectives of those most closely affected by the implementation were meaningfully incorporated in the evaluation. In the context of the ISCM evaluation, the thoughtful contributions and experiential insights of front-line staff and youth advisors substantially reshaped the drafted effectiveness principles. This resulted in a clarification of language, concepts, and expected outcomes for clients and service providers associated with each effectiveness principle, as well as each stakeholder's informal assessments of the evaluability of each principle from a client, provider, and system-level perspective. In this way, the evaluation process can facilitate multidirectional exchanges of knowledge that both support the consolidation of the effectiveness principles and contribute a deeper understanding of the emerging theories.

Second, the inclusion of front-line leaders and patient/client partner advisors in crafting effectiveness principles helped to communicate that the evaluation team, as well as the leadership of each initiative, is committed to an evolving, responsive, and contextualized understanding of the work being undertaken. This helps to build the relationship between the evaluation team and the intended users of the evaluation, ensuring that the users are comfortable in sharing their emerging insights of the implementation process and ultimately contributing to the utility of the evaluation.

GUIDE concludes with

Evaluable: Finally, PF-DE focuses on the principles as the evaluand, assessing how meaningful the principle is within each context, the extent to which these principles are used to understand and navigate each context as well as how well the outcomes that result from applying the principles align with the theorized outcomes. Therefore, integrating the process of drafting effectiveness principles with identifying indicators and data sources for assessing the effectiveness principles can be beneficial.

To support this process, we found it useful to consolidate effectiveness principles within an evaluation matrix. For example, in the ISCM evaluation, the matrix format allowed us to match specific indicators and data sources with each of the candidate effectiveness principles in an integrated and organized way. As a direct result of the matrix creation, Foundry's internal evaluation lead was able to revise Foundry's surveys to include specific dimensions related to our candidate effectiveness principles. Therefore, this process can also help to facilitate the 
participatory aspect of creating the effectiveness principles as the matrix can be completed collaboratively with stakeholders.

In addition, applying systems thinking to the selection and crafting of candidate principles helps to draw attention to the interconnected aspects of the initiative that are relevant to success but are outside the immediate focus of stakeholders. Depending on the context and the needs of the evaluation, we found that systems thinking can be incorporated in the effectiveness principles by drafting them as holistic and mutually reinforcing or integrated and sequential (where adherence to prior principles can facilitate the contexts required for subsequent principles). In the Megamorphosis evaluation, we used an integrated and sequential approach to drafting the effectiveness principles. For example, by applying the principle "Cultivate and sustain processes for team-reflection and continuous improvement," we theorized that teams would then be able to "generate and test solutions through shared governance by involving those who live, visit and work at each neighborhood." Consequently, the effectiveness principles were able to capture the interrelated and interdependent nature of the Megamorphosis initiative.

\section{Stakeholder review and finalization of effectiveness principles using GUIDE}

Once the effectiveness principles have been identified, they can be reviewed through a consultative process with relevant stakeholders using the GUIDE framework (Patton, 2018). This ensures that effectiveness principles have been framed in way that is useful, culturally and contextually appropriate, and evaluable. In the three evaluations presented in this paper, consultations were done through iterative workshops lasting between 60 and 90 minutes. Feedback on the candidate effectiveness principles was collected with Microsoft Excel templates (Table 2). The feedback informed an iterative process of adjusting and adapting the effectiveness principles until they are consolidated and agreed upon. Through this iterative process, metrics for assessing each effectiveness principle can also be established.

\section{LESSONS LEARNED}

Although developing effectiveness principles is a rigorous and resource-intensive process, it can help health-care teams to clearly define and articulate the principles that guide their values-driven and action-oriented programs or initiatives. The described systematic process leverages deep and trusting relationships with stakeholders that need to be established early in the evaluation to foster their understanding of the need for effectiveness principles. Flexibility in approaches is also essential. Time and resource constraints, challenges working with diverse groups with varied interests, as well as preformed principles and perspectives may require the evaluation team to proceed through these stages in a non-linear manner. Evaluators may also need to tactfully challenge pre-existing perspectives among the initiative team with emerging evidence to create the effectiveness 
principles. Finally, it is important for the evaluator to utilize a combination of systems and participatory approaches to ensure that all important elements, which may not seem obvious from the outset, are considered in the creation of effectiveness principles.

\section{CONCLUSION}

Crafting clearly articulated and meaningful effectiveness principles is a critical first step in setting up a principles-focused developmental evaluation, especially in complex dynamic contexts that exist in health-care service delivery systems. This process can foster a shared understanding of the evaluation between the team and the evaluator. However, to be beneficial, the effectiveness principles should be identified early in the evaluation through a collaborative process that ensures full engagement and the utility of evaluation findings and recommendations.

\section{ACKNOWLEDGEMENTS}

Our team gratefully acknowledges the support of Providence Health Care, St. Paul's Foundation, and the Michael Smith Foundation for Health Research for funding our evaluation work, as well as the Centre for Health Evaluation and Outcome Sciences for their ongoing support of our team. We would also like to thank the staff, leadership, and various teams from the evaluation sites for their time and participation. Finally, we would like to thank Dr. Chris Lovato (University of British Columbia) and Dr. Beth Snow (CHEOS and Simon Fraser University) for their critical reviews of this article.

\section{REFERENCES}

Bower, P., \& Gilbody, S. (2005). Stepped care in psychological therapies: Access, effectiveness and efficiency. British Journal of Psychiatry, 186(1), 11-17. https://doi. org/10.1192/bjp.186.1.11

Centers for Disease Control and Prevention. (2011, October). Self-study guide: Program evaluation. Program Performance and Evaluation Office (PPEO).

Cole, D. C., Aslanyan, G., Dunn, A., Boyd, A., \& Bates, I. (2014). Dilemmas of evaluation: Health research capacity initiatives. Bulletin of the World Health Organization, 92(12), 920-921. https://doi.org/10.2471/blt.14.141259

Dozois, E., Langlois, M., \& Blanchet-Cohen, N. (2010). A practitioner's guide to developmental evaluation. International Institute for Child Rights and Development.

Kinarsky, A. R., Alkin, M. C., \& Christie, C. A. (2019). What have we learned? New Directions for Evaluation, 2019(163), 83-93. https://doi.org/10.1002/ev.20372

Lambert, E. Y., Rebecca, S., Ashery, D. S. W., Richard, H., \& Needle, M. P. H. (1995). Qualitative methods in drug abuse and HIV research. Public Health Service National Institutes of Health National Institute on Drug Abuse Division of Epidemiology and Prevention Research. 
Miles, A., \& Asbridge, J. (2013). Contextualizing science in the aftermath of the evidencebased medicine era: On the need for person-centered healthcare. European Journal for Person Centered Healthcare, 1(2), 285. https://doi.org/10.5750/ejpch.v1i2.827

Patton, M. Q. (2011). Developmental evaluation: Applying complexity concepts to enhance innovation and use. Guilford Press.

Patton, M. Q. (2016). What is essential in developmental evaluation? On integrity, fidelity, adultery, abstinence, impotence, long-term commitment, integrity, and sensitivity in implementing evaluation models. American Journal of Evaluation, 37(2), 250-265. https://doi.org/10.1177/1098214015626295

Patton, M. Q. (2018). Principles-focused evaluation: The guide. Guilford Press.

Patton, M. Q., Mckegg, K., \& Wehipeihana, N. (Eds.). (2015). Developmental evaluation exemplars: Principles in practice. Guilford Press.

Salmon, A., Fernando, S., \& Berger, M. (2018). Developmental evaluation of Foundry's proof of concept.

Turner, R. E., \& Cromhout, P. B. (2020). Case study used to guide the development of effectiveness principles for Principles-Focused Evaluation. African Evaluation Journal, 8(1), 1-6. https://doi.org/10.4102/aej.v8i1.416

Wolfe, S. M., Long, P. D., \& Brown, K. K. (2020). Using a principles-focused evaluation approach to evaluate coalitions and collaboratives working toward equity and social justice. New Directions for Evaluation 165, 45-65. https://doi.org/10.1002/ev.20404

\section{AUTHOR INFORMATION}

Ihoghosa Iyamu is a project coordinator at the Centre for Health Evaluation and Outcome Sciences. He is a physician with over six years' experience in operations research, monitoring, and evaluation, spanning various health-related fields. He is also a graduate student of the University of British Columbia's School of Population and Public Health (SPPH) PhD program.

Mai Berger is a project coordinator at the Centre for Health Evaluation and Outcome Sciences. Her work in evaluation mainly encompasses youth-focused mental health and substance-use programs, community-based participatory programs, and knowledge translation. She is also a Master of Science student at the University of British Columbia's School of Population and Public Health.

Erika Ono is an evaluation specialist at the Centre for Health Evaluation and Outcome Sciences. She is also a PhD candidate in the School of Social Work, University of British Columbia.

Amy Salmon is a clinical associate professor at the UBC School of Population and Public Health; the Associate Director, Academic, Community, and Systems Engagement, at the Centre for Health Evaluation and Outcome Sciences (CHÉOS); and a research scientist and the Program Head - Knowledge Translation at CHÉOS. She has over 15 years of experience as a program evaluator in health systems. 